\section{Synthesis and Crystal Structure of $\mathrm{Ag}_{2} \mathrm{MnSnS}_{4}$}

\section{Sebastian Greil ${ }^{[a]}$ and Arno Pfitzner*[a]}

Keywords: Keywords:Tetrahedral compounds, quaternary silversulfides, wurtzite structure type

The tetrahedral compound $\mathrm{Ag}_{2} \mathrm{MnSnS}_{4}$ was synthesized using high-temperature solid-state synthesis in evacuated quartz-ampoules. The structure was solved using single-crystal X-ray diffraction. $\mathrm{Ag}_{2} \mathrm{MnSnS}_{4}$ crystallizes in the monoclinic spacegroup $P n$ with $R 1=3.48 \%$ and $w R^{2}=7.32 \%$ (all data). The lattice constants are $a=6.696(1) \AA, b=6.991(1) \AA, c=$ 8.222(2) $\AA$ and $\beta=90.00(3)^{\circ}$ (pseudo-orthorhombic). $\mathrm{Ag}_{2} \mathrm{MnSnS}_{4}$ can be derived from wurtzite via cross-substitution as shown by Parthé. ${ }^{[1]}$

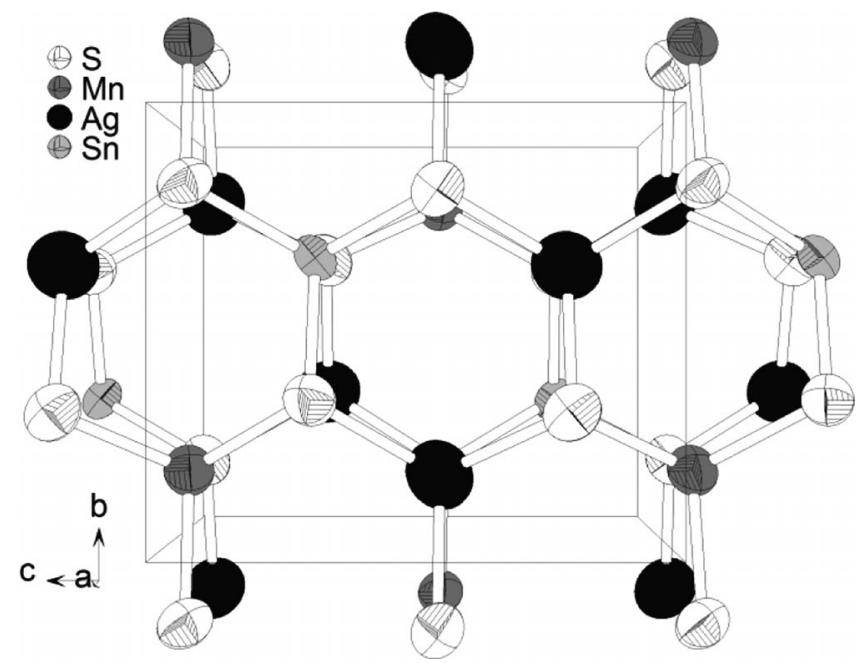

Figure 1. Structure of $\mathrm{Ag}_{2} \mathrm{MnSnS}_{4}$ viewed along the $a$-axis

[1] E. Parthé, in Crystal structures of intermetallic compounds (Eds.: J.H. Westbrook, R. L. Fleischer), J. Wiley \& Sons New York, 2000, Vol. 2, pp. 10-20.

\section{Elektrochemische Charakterisierung eines $\mathrm{Ce}_{0,8} \mathrm{Y}_{0,2} \mathrm{O}_{2-\delta}$-Einkristalls}

\author{
Maximilian Daniels, ${ }^{[a]}$ Gregor Ulbrich, ${ }^{[b]}$ \\ Hans-Dieter Wiemhöfer, ${ }^{[a]}$ and Martin $\operatorname{Lerch}^{[b]}$
}

Keywords: cerium oxide, mixed conductivity, single crystal

Durch gezielte Kationensubstitution von Cerdioxid können Eigenschaften wie z.B. Sauerstoff-ionenleitfähigkeit, Elektronenleitfähigkeit und auch Sauerstoffspeicherfähigkeit modifiziert werden. Um den Einfluss niedervalenter Kationen zu untersuchen, wurden makroskopische $\mathrm{Ce}_{0,8} \mathrm{Y}_{0,2} \mathrm{O}_{2-\delta}$-Einkristalle mithilfe der Skull-Schmelz-Technik ${ }^{[1]}$ gezüchtet. Diese Kristalle wurden mittels XRD, WDX und EBSD strukturell bzw. chemisch analysiert. Es liegt eine Fluorit-Struktur mit statistisch verteilten Kationen vor. Die elektrische Gesamtleitfähigkeit wurde mittels Impedanzspektroskopie untersucht, wohingegen für die Bestimmung der elektronischen Teilleitfähigkeit die Hebb-Wagner-Methode durch Mikrokontaktmessungen $^{[2]}$ zum Einsatz kam. Die Ionenleitfähigkeit von $\mathrm{Ce}_{0,8} \mathrm{Y}_{0,2} \mathrm{O}_{2-\delta}$ ist gegenüber reinem $\mathrm{CeO}_{2}$ erhöht, die elektronische Leitfähigkeit bleibt konstant.

[1] W. Assmus, N. Whippey, Chem. Ing. Technol. 1983, 55, 716-717.

[2] K. Schmale, Phys. Status Solidi B 2010, 1-9.

\footnotetext{
* Prof. Dr. A. Pfitzner

E-Mail: Arno.Pfitzner@chemie.uni-regensburg.de

[a] Institut für Anorganische Chemie, Universität Regensburg,

Universitätsstr. 31, 93053 Regensburg, Germany
}

\footnotetext{
* M. Daniels

E-Mail: max.daniels@uni-muenster.de

[a] Institut für anorganische und analytische Chemie, Westfälische Wilhelms-Universität Münster, Corrensstr. 28/30, 48149 Münster, Germany

[b] Institut für Chemie, Technische Universität Berlin, Straße des 17. Juni 135, 10623 Berlin, Germany
} 